\title{
Sleep and Sleep Homeostasis in Mice Lacking the 5-HT2c Receptor
}

\author{
Marcos G. Frank, Ph.D., Michael P. Stryker, Ph.D., and Laurence H. Tecott, M.D., Ph.D.
}

Studies in humans and rats indicate that serotonin (5-hydroxytryptamine, 5-HT) receptors are involved in mammalian sleep expression. We investigated the contribution of the 5-HT2c receptor to sleep expression by examining sleep patterns in mice bearing a targeted null mutation of this receptor. 5-HT2c receptor knock-out mice had more wakefulness, several abnormalities in rapid eye movement sleep expression and an enhanced response to sleep deprivation compared with wild-type control mice. These findings suggest that $5 \mathrm{HT} 2 \mathrm{c}$ receptors may mediate several effects on sleep that have been ascribed to serotonin. [Neuropsychopharmacology 27:869-873, 2002] (C) 2002 American College of Neuropsychopharmacology. Published by Elsevier Science Inc.
KEY WORDS: 5-HT; Sleep; Sleep deprivation; Knock-out; Transgenic; NonREM

Serotonin (5-HT) 2 receptors are thought to play an important role in the expression of both REM (rapid eye movement) and nonREM sleep (Dugovic and Wauquier 1987; Sharpley et al. 1994; Benington and Heller 1995). Antagonists with mixed affinity for 5-HT2c and 5-HT2a receptors (5-HT2cR, 5-HT2aR) increase the deeper stages of nonREM sleep and nonREM sleep electroencephalographic slow wave activity (EEG SWA) (Dugovic and Wauquier 1987; Sharpley et al. 1994; Benington and Heller 1995). In addition, pharmacological blockade of 5-HT2 receptors reduces REM sleep time and transitions from nonREM to REM sleep (NRT). These results suggest that 5-HT2 receptors may be involved in the homeo-

From the W. M. Keck Foundation Center for Integrative Neuroscience and Department of Physiology (MGF, MPS), and Department of Psychiatry (LHT), University of California, San Francisco, CA.

Address correspondence to: Marcos G. Frank, Ph.D., Department of Physiology-UCSF, 513 Parnassus Avenue, University of California, San Francisco, CA 94143-0444. Tel.: (415) 476-1311; Fax: (415) 476-4929; E-mail: mgf@phy.ucsf.edu

Received February 24, 2002; revised April 10, 2002; accepted April 23, 2002.

Online publication: 4/26/02 at www.acnp.org/citations/ Npp042602296. static regulation of nonREM sleep and the timing of the nonREM-REM sleep cycle.

Determining the precise role of the 5-HT2aR and 5 -HT2cR in sleep has proven difficult because both serotonin receptor sub-types couple to similar intracellular mechanisms (Julius et al. 1990) and most pharmacological compounds used to examine the function of 5-HT2 receptors have affinity for both the 5-HT2aR and 5-HT2cR (Sharpley et al. 1994), as well as for some adrenergic and histaminergic receptors (Monti and Monti 1999). The development of mice with targeted mutations of receptor genes provides an alternative approach for examining the contribution of a given serotonin receptor to sleep expression (Boutrel et al. 1999). In order to test the hypothesis that 5-HT2cRs are involved in sleep expression, we examined sleep and sleep homeostasis in mice bearing a targeted null mutation of the 5-HT2cR.

\section{METHODS}

\section{5-HT2cR Mutant Mice}

These animals were originally generated from a 129derived embryonic stem cell line, and were subsequently backcrossed for at least ten generations to a C57BL/ 6 background. Genotyping was performed according to 
previously described methods (Tecott et al. 1998). 5-HT2cR knock-out $(\mathrm{KO})$ mice appear healthy, breed readily and exhibit no overt abnormalities in central nervous system morphology or expression levels of other 5-HT receptor subtypes (Tecott et al. 1998; López-Giménez et al. 2001).

\section{Surgeries, Housing, and Vigilance State Recording}

Male wild-type (WT: $\mathrm{n}=8$ ) and 5-HT2cR knockout mice (KO: $n=7$ ) between two and three months of age were implanted with EEG and EMG electrodes under isoflorane anesthesia. After 5-7 days of post-operative recovery, lightweight recording cables were connected to the head implants and mice were placed in cylindrical polypropylene containers containing nest material, water and food ad libitum. Mice were acclimated to the sleep chamber for 5-7 days (12:12 light-dark (LD) cycle; lights on at 8 A.M.). EEGs and EMGs were then collected on a Grass 7 polygraph, digitized at $100 \mathrm{~Hz}$ and stored in 10-s epochs on a personal computer according to previously described methods (Frank and Heller 1997). Following acclimation, baseline 24-h recordings were made. All surgical and sleep recording procedures were approved by the UCSF Committee for Animal Research and were performed in accordance with the Declaration of Helsinki and the National Institutes of Health Guide for the Care and Use of Laboratory Animals.

\section{Vigilance State Scoring and Analyses}

NonREM sleep, REM sleep, and wake were determined by an experimenter blind to mice genotypes using an algorithm validated in mice (Veasey et al. 2000). On the baseline day, we computed the amount of each state (expressed as a percentage of total recording time) and the duration of individual sleep and wake episodes. We also measured transitions between nonREM sleep and REM sleep during the light phase. Since 5-HT2 receptor antagonists are reported to produce abnormal EEGs in rodents (Borbely et al. 1988), we also examined differences in vigilance state EEGs according to previously described methods (Frank and Heller 1997).

To investigate sleep homeostasis in 5-HT2cR KO mice, we sleep deprived (SD) mice from 8 A.M. to 2 P.M. at the beginning of the major sleep period (light phase) using a slowly rotating chamber. Vigilance state amounts and durations in the remaining $6 \mathrm{~h}$ of the light phase and subsequent $12 \mathrm{~h}$ of the dark phase (POST-SD) were compared with mean values obtained from timematched periods of the corresponding baseline (PRE$\mathrm{SD})$. The amount of nonREM sleep SWA in 2-h bins (normalized to baseline 24-h nonREM sleep SWA) in the remaining half of the light phase and subsequent dark phase was likewise compared with time-matched values obtained in the baseline light and dark periods.

\section{Statistical Analyses}

General Linear Model tests (GLM: ANOVAs for unbalanced cells in multiple hour comparisons) were used to test between baseline WT and 5-HT2cR KO values when more than two comparisons were made. Protected $t$-tests (Student-Newman-Keuls: SNK) were used for further tests between values when significant main (M) or interaction $(X)$ effects were obtained in the GLM. Student's $t$-tests were used for all other comparisons. Statistics were calculated using SAS statistical software (6.03 edition).

\section{RESULTS}

\section{Baseline Sleep}

We found that 5-HT2cR KO and WT mice differed on several sleep measures in the baseline (PRE-SD) period. 5-HT2cR KO mice had less nonREM sleep ( $\mathrm{F}=13.46, p<$ $.0008)$ and more wake $(\mathrm{F}=11.14, p<.002)$ than WT mice, particularly in the dark phase of the 24-h day (Figure 1, panel A). Individual waking bouts were also significantly longer in the 5 -HT2cR KO mice $(\mathrm{F}=6.1, p<.02$, Figure 1 , panel B). 5-HT2cR KO mice also had fewer NRTs per hour of nonREM sleep than WT mice (mean and SEM: KO 11.6 \pm 0.8 , WT $14.5 \pm 0.8$; Student's $t$-test, $p<.05$ ), but on average, each NRT in 5-HT2cR KO mice was more likely to be followed by a sustained ( $>30 \mathrm{~s}$ ) REM sleep period than for WT mice (mean and SEM: 5-HT2cR KO 40.5\% $\pm 3.3 \%$, WT $30.9 \% \pm 2.6 \%$; Student's $t$-test, $p<.05$ ). There were, however, no differences in vigilance state EEGs between WT and 5-HT2cR KO mice (Figure 2, GLM, $p>$.05).
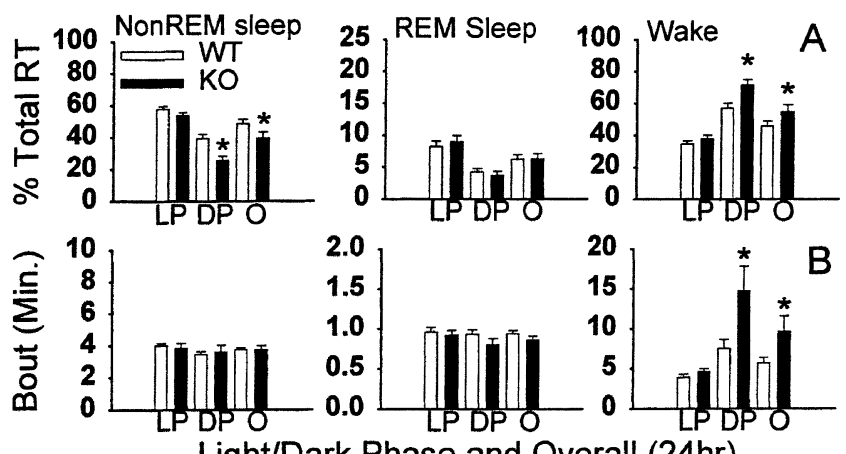

Light/Dark Phase and Overall (24hr)

Figure 1. Vigilance state amounts and duration in wild-type (WT) and 5-HT2cR knock-out (KO) mice. A. Mean ( \pm SEM) amounts (expressed as a \% of total recording time (RT)) of nonREM sleep, REM sleep and wake are shown for the light phase (LP: 8 A.M.-8 P.M.) dark phase (DP: 8 P.M. -8 A.M.), and overall (O: $24 \mathrm{~h})$ in the baseline period. B. Mean ( \pm SEM) duration (in minutes (Min.)) of nonREM sleep, REM sleep, and wake bouts shown for the LP, DP portions and overall (O) of the baseline period. * Significant difference between the groups (GLM, SNK, $p<.05)$. 


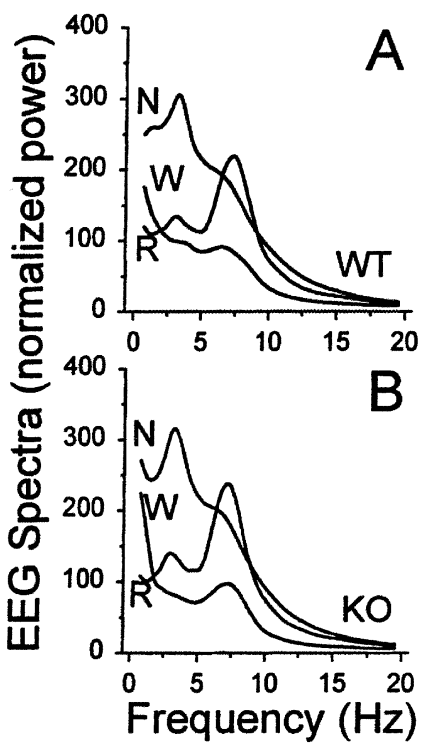

Figure 2. Mean vigilance state EEG spectra in (A) wildtype (WT) and (B) 5-HT2cR knock-out (KO) mice. EEG spectral power in each vigilance state is expressed as a percentage of the mean power in all EEG frequencies averaged across all vigilance states (normalized power). There were no significant differences in EEG spectra in nonREM (N) sleep, REM (R) sleep, and wake (W) between the groups.

\section{Effects of 6-h SD}

Sleep deprivation produced greater homeostatic responses in 5-HT2c KO mice than in WT mice as measured by changes in nonREM sleep SWA (Figure 3), episode duration and nonREM sleep time (Figure 3, Table 1). These different responses to SD were not due to phenotypic differences in arousal during the SD. Both groups of mice were mostly awake during the SD (mean wake and SEM: $5-\mathrm{HT} 2 \mathrm{cR} \mathrm{KO} \mathrm{98.7 \%} \mathrm{\pm} \mathrm{3.8 \% ,} \mathrm{WT} \mathrm{93.2 \%} \pm 0.6 \%$ total RT: Student's t-test, $\mathrm{p}>.05)$, and the amount of EEG SWA (expressed as \% of baseline nonREM sleep values) during sleep deprivation at low levels relative to baseline nonREM sleep values (mean and SEM: 5-HT2cR KO 33\% \pm $1.9 \%$, WT $41.3 \% \pm 3.8 \%$ : Student's t-test, $\mathrm{p}>.05$ ).

\section{DISCUSSION}

To test the hypothesis that 5-HT2cRs are involved in sleep expression, we examined sleep architecture and homeostasis in a line of mutant mice devoid of functional 5-HT2cR. We found several abnormalities in sleep/wake architecture and an enhanced response to sleep deprivation in 5-HT2cR KO mice. These results suggest that the 5-HT2cR contributes significantly to the serotonergic regulation of sleep.

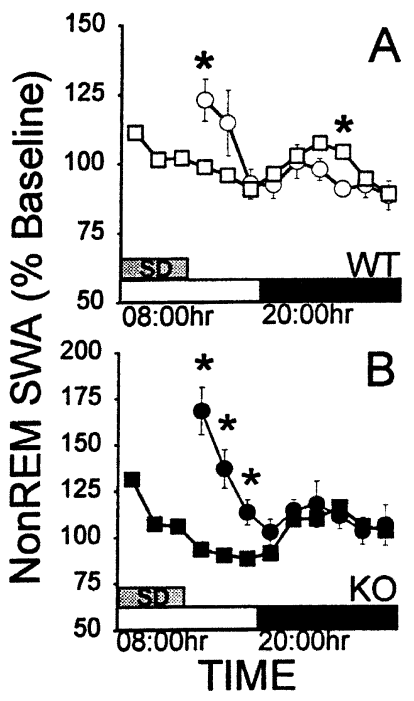

Figure 3. Effects of 6-h sleep deprivation (SD) on nonREM sleep EEG slow wave activity (SWA) in (A) wild-type (WT) and (B) 5-HT2cR KO mice. Mean ( \pm SEM) nonREM sleep SWA values following sleep deprivation (POST-SD: circles) and in the PRE-SD period (squares) are expressed as a $\%$ of the mean 24-h nonREM SWA values obtained in the baseline day of sleep/wake recording. "Significant difference between PRE-SD and POST-SD values (5-HT2cR KO: PRE-SD vs. POSTSD X Hour: $\mathrm{F}=4.42, p<.0001$, SNK, $p<.05$; WT:PRE-SD vs. POST-SD X Hour: $\mathrm{F}=2.11, p<.04$, SNK, $p<.05$ ).

\section{Sleep Architecture in 5-HT2cR KO Mice}

Mice lacking the 5-HT2cR had several abnormalities of sleep/wake architecture. Compared with WT mice, 5-HT2cR KO mice had greater amounts and longer episodes of wake (with a corresponding lower amount of nonREM sleep) and fewer nonREM-REM transitions. These results indicate that 5-HT2cRs are involved in basal regulation of wake and nonREM sleep amounts and suggest that 5-HT2cRs influence the nonREM-REM sleep cycle.

\section{Sleep Homeostasis and the 5-HT2cR}

Sleep is regulated by homeostatic mechanisms that control the intensity and amount of sleep as a function of prior sleep/wake history (reviewed in Dijk and Edgar 1999). Our findings of an enhanced response to sleep deprivation in 5-HT2cR KO mice suggest that the 5 -HT2cR may play an important role in this process. Although this enhanced response to sleep deprivation may in part be due to more wake in 5-HT2cR KO mice during the dark phase prior to the SD (5-HT2cR KO: 8.52 $\mathrm{h}$; WT: $6.8 \mathrm{~h}$ ), it may also reflect alterations in the accumulation of sleep propensity. For example, the enhanced response to sleep loss in 5-HT2cR KO mice may be related to increased dopamine and noradrenaline ac- 
Table 1. Effects of 6-h Sleep Deprivation (SD) on Wild-type (WT) and 5HT2cR Knock-out (KO) Mice Vigilance State Amounts and Bout Duration.

\begin{tabular}{|c|c|c|c|c|c|c|c|c|}
\hline & \multicolumn{4}{|c|}{ Light Phase } & \multicolumn{4}{|c|}{ Dark Phase } \\
\hline & \multicolumn{2}{|c|}{ Recording Time } & \multicolumn{2}{|c|}{ Bout Duration } & \multicolumn{2}{|c|}{ Recording Time } & \multicolumn{2}{|c|}{ Bout Duration } \\
\hline & Pre-SD & Post-SD & Pre-SD & Post-SD & Pre-SD & Post-SD & Pre-SD & Post-SD \\
\hline \multicolumn{9}{|l|}{$W T$} \\
\hline NonREM & $58.2 \pm 2.3$ & $59.8 \pm 3.1$ & $3.9 \pm 0.2$ & $4.7 \pm 0.3$ & $42.5 \pm 2.2$ & $41.5 \pm 2.3$ & $3.4 \pm 0.3$ & $3.4 \pm 0.2$ \\
\hline REM & $7.2 \pm 0.6$ & $8.0 \pm 0.9$ & $0.8 \pm 0.1$ & $0.9 \pm 0.1$ & $4.7 \pm 0.4$ & $5.6 \pm 0.5$ & $0.8 \pm 0.1$ & $0.8 \pm 0.1$ \\
\hline Wake & $34.6 \pm 2.6$ & $32.2 \pm 3.5$ & $4.2 \pm 0.5$ & $3.9 \pm 0.5$ & $52.8 \pm 2.5$ & $52.9 \pm 2.7$ & $6.7 \pm 0.8$ & $7.5 \pm 0.9$ \\
\hline \multicolumn{9}{|l|}{$\mathrm{KO}$} \\
\hline NonREM & $51.1 \pm 3.1$ & $62.5 \pm 2.3^{*}$ & $3.5 \pm 0.2$ & $6.3 \pm 0.6^{*}$ & $26.2 \pm 2.7$ & $37.7 \pm 2.4^{*}$ & $2.7 \pm 0.2$ & $4.3 \pm 0.3^{*}$ \\
\hline REM & $7.4 \pm 0.9$ & $7.9 \pm 0.7$ & $0.9 \pm 0.1$ & $1.0 \pm 0.1$ & $3.9 \pm 0.6$ & $4.4 \pm 0.4$ & $0.5 \pm 0.1$ & $0.7 \pm 0.1$ \\
\hline Wake & $41.5 \pm 3.7$ & $29.6 \pm 3.2 *$ & $4.1 \pm 0.5$ & $3.9 \pm 0.7$ & $69.9 \pm 3.1$ & $57.9 \pm 2.8^{*}$ & $13 \pm 2.8$ & $10.5 \pm 1.9$ \\
\hline
\end{tabular}

Mean ( \pm SEM) vigilance state amounts (as \% total recording time) and state episode duration (in minutes) are displayed for baseline (PRE-SD) periods in light (2 P.M.-8 P.M.) and dark phase (8 P.M.-8 A.M.), and time-matched recovery periods (POST-SD) in the remaining half of the light phase and the subsequent dark phase.

${ }^{*}$ Indicates significant difference between POST-SD and PRE-SD values (Student's $t$-test, $p<0.05$ ).

tivity in these mutants. 5-HT2cRs tonically inhibit these catecholaminergic systems (Wright et al. 1995; Di Matteo et al. 1999; Gobert et al. 2000). Catecholaminergic hyperactivity can in turn induce hypervigilance and large compensatory changes in sleep expression (Edgar et al. 1995; Touret et al. 1995; Caldwell and Caldwell 1997). It is therefore possible that enhanced catecholaminergic neurotransmission in 5-HT2cR KO mice results in a more rapid accumulation of sleep propensity.

\section{ACKNOWLEDGMENTS}

NIH Grant EY02874 and National Research Service Awards EY60831 and EY06880 supported this research.

\section{REFERENCES}

Benington JH, Heller HC (1995): Monoaminergic and cholinergic modulation of REM-sleep timing in rats. Brain Res 681:141-146

Borbely AA, Trachsel L, Tobler I (1988): Effects of ritanserin on sleep stages and sleep EEG in the rat. Eur J Pharmacol 156:275-278

Boutrel B, Franc B, Hen R, Hamon M, Adrien J (1999): Key role of $5 \mathrm{ht} 1 \mathrm{~b}$ receptors in the regulation of paradoxical sleep as evidenced in 5ht1b knock-out mice. J Neurosci 19:3204-3212

Caldwell JL, Caldwell JA (1997): Recovery sleep and performance following sleep deprivation with dextroamphetamine. J Sleep Res 6:92-101

Dijk DJ, Edgar DM (1999): Circadian and homeostatic control of wakefulness and sleep. In Turek FW, Zee PC (eds), Regulation of Sleep and Circadian Rhythms, Vol 133. New York, Marcel Dekker Inc., pp 111-148

Di Matteo V, Di Giovanni G, Di Mascio M, Esposito E (1999): SB 242084, a selective serotonin2C receptor antagonist, increases dopaminergic transmission in the mesolimbic system. Neuropharmacology 38:1195-1205
Dugovic C, Wauquier A (1987): 5HT2 receptors could be primarily involved in the regulation of slow-wave sleep in the rat. Eur J Pharmacol 137:145-146

Edgar DM, Seidel WF, Contreras PC, Dement WC (1995): Wakefulness, motor activity, and compensatory sleep responses following caffeine, methamphetamine, and modafinil treatment in rats. Sleep Res 24A:21

Frank MG, Heller HC (1997): Neonatal treatments with the serotonin uptake inhibitors clomipramine and zimelidine, but not the noradrenaline uptake inhibitor desipramine, disrupt sleep patterns in adult rats. Brain Res 768:287-293

Gobert A, Rivet JM, Lejeune F, Newman-Tancredi A, Adhumeau-Auclair A, Nicolas JP, Cistarelli L, Melon C, Millan MJ (2000): Serotonin(2C) receptors tonically suppress the activity of mesocortical dopaminergic and adrenergic, but not serotonergic, pathways: a combined dialysis and electrophysiological analysis in the rat. Synapse 36:205-221

Julius D, Huang KN, Livellie TJ, Axel R, Jessel TM (1990): The 5HT2 receptor defines a family of structurally distinct but functionally conserved serotonin receptors. Proc Natl Acad Sci USA 87:928-932

López-Giménez JF, Tecott LH, Palacios JM, Guadalupe M, Vilaró MT (2001): Serotonin 5-HT2C receptor knockout mice: autoradiographic analysis of multiple serotonin receptors. J Neurosci Res 67(1):69-85

Monti JM, Monti D (1999): Functional role of serotonin $5-\mathrm{HT} 1$ and 5-HT2 receptor in the regulation of REM sleep. In Mallick BN, Inoue S (eds), Rapid Eye Movement Sleep. New Delhi, Narosa Publishing House, pp 142-152

Sharpley AL, Elliot JM, Attenburrow MJ, Cowen PJ (1994): Slow wave sleep in humans: role of 5HT2a and 5HT2c receptors. Neuropharmacology 33:467-471

Tecott LH, Logue SF, Wehner JM, Kauer JA (1998): Perturbed dentate gyrus function in serotonin 5-HT2c receptor mutant mice. Proc Natl Acad Sci USA 95:15026-15031

Touret M, Sallanon-Moulin M, Jouvet M (1995): Awakening properties of modafinil without paradoxical sleep rebound: Comparative study with amphetamine in the rat. Neurosci Lett 189:43-46 
Veasey SC, Valladares O, Fenik P, Kapfhamer D, Sanford L, Benington J, Bucan M (2000): An automated system for recording and analysis of sleep in mice. Sleep 23:10251040
Wright DE, Seroogy KB, Lundgren KH, Davis BM, Jennes L (1995): Comparative localization of serotonin1A, 1C, and 2 receptor subtype mRNAs in rat brain. J Comp Neurol 351:357-373 